\title{
Affinity- and topology-dependent bound on current fluctuations
}

\author{
Patrick Pietzonka ${ }^{1}$, Andre C. Barato ${ }^{2}$, and Udo Seifert ${ }^{1}$ \\ ${ }^{1}$ II. Institut für Theoretische Physik, Universität Stuttgart, 70550 Stuttgart, \\ Germany \\ ${ }^{2}$ Max Planck Institute for the Physics of Complex Systems, Nöthnitzer Straße \\ 38, 01187 Dresden, Germany
}

\begin{abstract}
We provide a proof of a recently conjectured universal bound on current fluctuations in Markovian processes. This bound establishes a link between the fluctuations of an individual observable current, the cycle affinities driving the system into a non-equilibrium steady state, and the topology of the network. The proof is based on a decomposition of the network into independent cycles with both positive affinity and positive stationary cycle current. This formalism allows for a refinement of the bound for systems in equilibrium or with locally vanishing affinities.

Keywords: Current fluctuations, large deviations, cycle decomposition, enzyme kinetics

PACS numbers: 05.70.Ln, 05.40.-a
\end{abstract}

Introduction. A comprehensive mathematical description of systems driven into a non-equilibrium steady state (NESS) is an open challenge in statistical physics. So far, the arguably most prominent universal property of these systems is the fluctuation theorem, establishing a symmetry between the probability of current fluctuations and the corresponding sign-reversed fluctuations [1-4]. Recently, we have proposed bounds on the probability of current fluctuations, which hold universally in systems that can be represented in terms of continuous-time Markov networks [5]. These bounds are formulated within the mathematical framework of large deviation theory $[6,7]$. This theory describes the decay of the probability of current fluctuations with time $t$ as an exponential law

$$
p(J, t) \sim \mathrm{e}^{-t I(J)},
$$

where $J$ denotes a generic fluctuating current, e.g., a cycle current, a current along an individual transition, the current associated with the entropy production or even a vector of several such currents [5]. The non-negative and convex function $I(J)$ is called a rate function or large deviation function. A dual description of the exponential law (1) is given in terms of the scaled cumulant generating function

$$
\lambda(z) \equiv \lim _{t \rightarrow \infty} \frac{1}{t} \log \left\langle\mathrm{e}^{z J t}\right\rangle \equiv \lim _{t \rightarrow \infty} \frac{1}{t} \log \int \mathrm{d} J p(J, t) \mathrm{e}^{z J t}=\max _{J}[z J-I(J)],
$$


where the integral runs over all possible realizations of the current $J$. The function $\lambda(z)$ is the Legendre-Fenchel transform of $I(J)$. In Ref. [5], we have presented several upper bounds on $I(J)$ and respective lower bounds on $\lambda(z)$. In particular, a parabolic bound depends only on the average entropy production of the system. Recently, this bound was proven by Gingrich et al [8]. Building partially on the ideas in their proof, we prove a second, stronger bound that requires additional information on the affinities and the topology of the Markov network [5]. This bound is saturated for an asymmetric random walk driven by a single affinity, for which $\lambda(z)$ has the shape of a hyperbolic cosine. Remarkably, this shape persists as a bound for processes on finite networks with arbitrarily complex topology and non-uniform transition rates. Since by construction the bounds are saturated for the most likely, stationary value $J=J^{\mathrm{s}}$ with $I\left(J^{\mathrm{s}}\right)=0$ (or, respectively, for $z=0$ with $\lambda(0)=0$ and $\lambda^{\prime}(0)=J^{\mathrm{s}}$ ), they imply particularly strong bounds on the typical Gaussian fluctuations around $J^{\mathrm{s}}$, which have been previously conjectured in terms of the thermodynamic uncertainty relation [9] and an affinity dependent bound on the Fano factor [10]. In these forms, the bounds can be experimentally applied to, e.g., enzymatic reaction networks or small electronic circuits.

Setup. We consider a Markov process on a finite network of $N$ states $\{i\}$ with transition rates $k_{i j}$ from state $i$ to state $j$ and assume micro-reversibility, i.e., $k_{i j}>0$ implies $k_{j i}>0$. The stationary probability distribution $p_{i}^{\mathrm{s}}$, satisfying

$$
\sum_{j}\left(p_{i}^{\mathrm{s}} k_{i j}-p_{j}^{\mathrm{s}} k_{j i}\right)=0
$$

gives rise to the stationary currents

$$
J_{i j}^{\mathrm{s}} \equiv p_{i}^{\mathrm{s}} k_{i j}-p_{j}^{\mathrm{s}} k_{j i}=-J_{j i}^{\mathrm{s}} .
$$

These stationary currents are the expectation values of the fluctuating currents $J_{i j}$, defined as the net number of transitions in a stochastic trajectory along the edge $(i, j)$ divided by the observation time $t$. An individual transition $i \rightarrow j$ contributes [4]

$$
f_{i j} \equiv \log \frac{p_{i}^{\mathrm{s}} k_{i j}}{p_{j}^{\mathrm{s}} k_{j i}}
$$

to the entropy production in a NESS, leading to the average total entropy production

$$
\sigma \equiv \sum_{i<j} J_{i j}^{\mathrm{s}} f_{i j}
$$

We denote by $\sum_{i<j}$ the sum over those edges $(i, j)$ in the network graph for which transitions are possible, i.e., for which $k_{i j}>0$ and $k_{j i}>0$.

The decomposition of networks into cycles is an important concept providing a link between (bio-)physical properties of a system and its description as a stochastic process [11-13]. A cycle $\mathcal{C}_{\alpha}$ of length $n_{\alpha} \geq 3$ (smaller cycles are not relevant for our discussion) is defined as a directed, self-avoiding, closed path $[\ell(1) \rightarrow \ell(2) \rightarrow \cdots \rightarrow$ $\ell\left(n_{\alpha}\right) \rightarrow \ell(1)$ ] along edges of the network with $k_{\ell(n) \ell(n+1)}>0$. We define the directed adjacency matrix $\chi^{\alpha}=\left(\chi_{i j}^{\alpha}\right)$ of such a cycle as

$$
\chi_{i j}^{\alpha} \equiv \sum_{n=1}^{n_{\alpha}} \delta_{\ell(n), i} \delta_{\ell(n+1), j}-\delta_{\ell(n), j} \delta_{\ell(n+1), i},
$$


which is +1 for edges $(i, j)$ where $\mathcal{C}_{\alpha}$ passes in forward direction, -1 for the backward direction and zero otherwise. A set of cycles $\left\{\mathcal{C}_{\alpha}\right\}$ is called complete if any set of currents $\left\{J_{i j}\right\}$ along the edges of the network consistent with Kirchhoff's law

$$
\sum_{j} J_{i j}=0 \quad \text { for all } i
$$

can be decomposed into a set of cycle currents $\left\{\mathcal{J}_{\alpha}\right\}$ such that

$$
J_{i j}=\sum_{\alpha} \mathcal{J}_{\alpha} \chi_{i j}^{\alpha}
$$

In particular, the stationary currents $J_{i j}^{\mathrm{s}}$ can be decomposed into stationary cycle currents $\mathcal{J}_{\alpha}^{\mathrm{s}}$. The affinity $\mathcal{A}_{\alpha}$ of a cycle is given by

$$
\mathcal{A}_{\alpha} \equiv \sum_{i<j} \chi_{i j}^{\alpha} \log \frac{k_{i j}}{k_{j i}}=\sum_{i<j} \chi_{i j}^{\alpha} f_{i j}=\sum_{(i, j) \in \mathcal{C}_{\alpha}} f_{i j},
$$

which can be used to write the average entropy production (6) as

$$
\sigma=\sum_{\alpha} \mathcal{J}_{\alpha}^{\mathrm{s}} \mathcal{A}_{\alpha}
$$

Main result. With the above definitions at hand, we can now state the central result proven in this Letter. The scaled cumulant generating function

$$
\lambda(\boldsymbol{z}) \equiv \lim _{t \rightarrow \infty} \frac{1}{t} \log \left\langle\mathrm{e}^{\boldsymbol{z} \cdot \mathcal{J} t}\right\rangle
$$

for the fluctuations of the vector $\mathcal{J}=\left(\mathcal{J}_{\alpha}\right)$ of cycle currents is bounded from below by

$$
\lambda(\boldsymbol{z}) \geq \sigma \frac{\cosh \left[\left(\boldsymbol{z} \cdot \mathcal{J}^{\mathrm{s}} / \sigma+1 / 2\right) \mathcal{A}^{*} / n^{*}\right]-\cosh \left[\mathcal{A}^{*} /\left(2 n^{*}\right)\right]}{\left(\mathcal{A}^{*} / n^{*}\right) \sinh \left[\mathcal{A}^{*} /\left(2 n^{*}\right)\right]},
$$

where $\mathcal{A}^{*} / n^{*}$ is defined as the smallest positive value for the affinity per cycle length over all cycles in the network. In a scalar version, this bound implies for the scaled cumulant generating function (2) of any generic current $J$, which can be written as an expansion in the cycle currents, the bound

$$
\lambda(z) \geq \sigma \frac{\cosh \left[\left(z J^{\mathrm{s}} / \sigma+1 / 2\right) \mathcal{A}^{*} / n^{*}\right]-\cosh \left[\mathcal{A}^{*} /\left(2 n^{*}\right)\right]}{\left(\mathcal{A}^{*} / n^{*}\right) \sinh \left[\mathcal{A}^{*} /\left(2 n^{*}\right)\right]},
$$

where $J^{\mathrm{s}}$ is the stationary value of $J$. In particular, for the entropy current $\sum_{\alpha} \mathcal{J}_{\alpha} \mathcal{A}_{\alpha}$ we have $J^{\mathrm{s}}=\sigma$, which further simplifies (14). Based on strong numerical evidence we have conjectured the relation (13) in Ref. [5]. Furthermore, Eq. (13) generalizes a bound on the Fano factor in enzyme kinetics that has been conjectured in Ref. [10].

Uniform cycle decomposition. For multicyclic networks, the choice of a minimal complete set of cycles is not unique. We make use of this ambiguity to expand the stationary current $J_{i j}^{\mathrm{s}}$ in a specific set of cycles $\left\{\mathcal{C}_{\beta}\right\}$. This set is constructed in a way that every cycle $\mathcal{C}_{\beta}$ contributing to the stationary current, i.e., for which $\mathcal{J}_{\beta}^{\text {s }} \neq 0$, satisfies the following properties, which will turn out to be essential for our proof of the affinity dependent bound (13): 

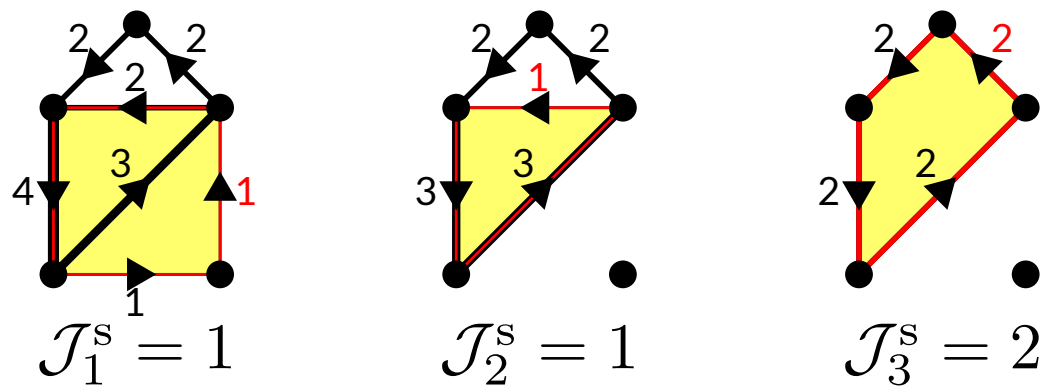

Figure 1. Example for a decomposition of a 5-state network into a complete set of three independent uniform cycles. Edges are labeled by the strength of the currents $J_{i j}^{(\beta)}$, whose direction is marked by arrows. For each of the three iteration steps, an edge with minimal current is labeled in red and the uniform cycle $\mathcal{C}_{\beta}$ including this edge is shown as a yellow polygon with red border.

(i) The edges of every cycle are aligned with the stationary current, i.e.,

$$
\operatorname{sgn} \chi_{i j}^{\beta}=\operatorname{sgn} J_{i j}^{\mathrm{s}}=\operatorname{sgn} f_{i j}
$$

for all $i, j$ and $\beta$. We call such cycles uniform with respect to $J_{i j}^{\mathrm{s}}$. The equality with $\operatorname{sgn} f_{i j}$ follows directly from the definitions (4) and (5) and shows via Eq. (10) that uniform cycles have positive affinity.

(ii) All stationary cycle currents are strictly positive, $\mathcal{J}_{\beta}^{\mathrm{s}}>0$ for all $\beta$.

It can be easily checked that an arbitrary cycle decomposition, for example the decomposition into fundamental cycles by Schnakenberg [11], does not necessarily meet these conditions. A construction of a cycle decomposition that satisfies at least condition (ii) was introduced by J. MacQueen [14], its physical relevance and proof is also discussed in Ref. [15]. This decomposition, however, refers only to networks with irreversible transitions. The network of our present setup with reversible transitions could be mapped on such a network by replacing every edge by two irreversible links with antiparallel direction, yet this procedure does not necessarily lead to a decomposition satisfying condition (i).

Here, we present a variation of the algorithm of MacQueen for networks with genuinely reversible transitions, which generates a set of cycles satisfying both conditions (i) and (ii), as exemplified in Fig. 1.

- Initialization. Set $J_{i j}^{(1)} \equiv J_{i j}^{\mathrm{s}}$.

- Iteration over $\beta \geq 1$. Locate an edge $\left(i^{*}, j^{*}\right)$ with minimal positive current $J_{i^{*} j^{*}}^{(\beta)}$ and set

$$
\mathcal{J}_{\beta}^{\mathrm{s}} \equiv \min _{i, j \mid J_{i j}^{(\beta)}>0} J_{i j}^{(\beta)}=J_{i^{*} j^{*}}^{(\beta)} .
$$

Construct $\mathcal{C}_{\beta}$ as a closed self avoiding path starting with the edge $\left(i^{*}, j^{*}\right)$ and passing only along edges in the direction for which $J_{i j}^{(\beta)}>0$. This path is not necessarily unique, one of several such paths can be chosen freely as $\mathcal{C}_{\beta}$. Using the directed adjacency matrix $\chi_{i j}^{\beta}$ corresponding to $\mathcal{C}_{\beta}$, as defined in Eq. (7), set

$$
J_{i j}^{(\beta+1)}=J_{i j}^{(\beta)}-\mathcal{J}_{\beta}^{\mathrm{s}} \chi_{i j}^{\beta} .
$$


a)

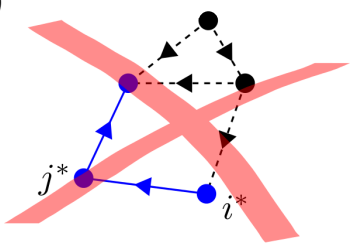

b)

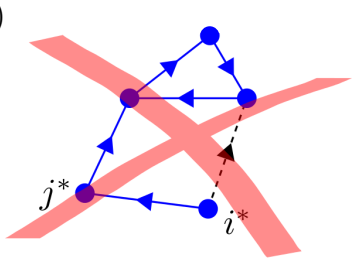

c)

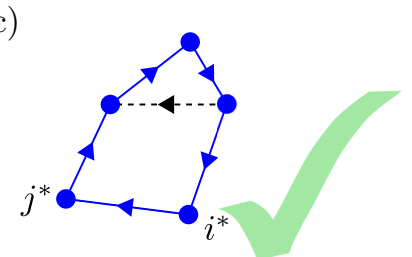

Figure 2. Illustration of a uniform cycle $\mathcal{C}_{\beta}$ starting with the edge $\left(i^{*}, j^{*}\right)$. The direction of $J_{i j}^{(\beta)}$ is indicated by the arrows, the (attempted) cycle is shown as solid blue lines. Neither dead ends (a) nor "dead-cycles" (b) are consistent with Kirchhoff's law (8).

- Terminate when $J_{i j}^{(\beta+1)}=0$ for all $i, j$.

By construction, the currents $J_{i j}^{(\beta)}$ satisfy Kirchhoff's law (8) for every $\beta$. As a consequence, it is always possible to construct an appropriate uniform cycle $\mathcal{C}_{\beta}$ following the direction of $J_{i j}^{(\beta)}$ : For every node where there is a way in, there must also be a way out, which rules out dead ends (see Fig. 2a). Similarly, it is not possible to run into a cycle without exit that does not include the starting node $i^{*}$ (see Fig. 2b). On a finite set of states, every self avoiding path must reach the starting point $i^{*}$ again after at most $N$ steps. Typically, there is more than one cycle starting with $\left(i^{*}, j^{*}\right)$ meeting these conditions. Any of these cycles can be selected as $\mathcal{C}_{\beta}$. Since we use in every iteration the minimal current as new cycle current, the individual currents $J_{i j}^{(\beta+1)}$ are either zero or have the same sign as $J_{i j}^{\mathrm{s}}$. Thus, every cycle that is uniform with respect to the currents $J_{i j}^{(\beta)}$ is also uniform with respect to $J_{i j}^{\mathrm{s}}$, as required by condition (i). The matrices $\chi_{i j}^{\beta}$ are linearly independent, since for every $\beta$ the edge $\left(i^{*}, j^{*}\right)$ is no longer contained in all the subsequent cycles. By construction, the algorithm leads to the decomposition

$$
J_{i j}^{\mathrm{s}}=\sum_{\beta} \mathcal{J}_{\beta}^{\mathrm{s}} \chi_{i j}^{\beta} .
$$

Typically, the algorithm terminates when $\beta$ reaches the number of fundamental cycles. It cannot terminate later since all cycles $\mathcal{C}_{\beta}$ are linearly independent. It may happen that the algorithm terminates earlier, so that the set of cycles $\left\{\mathcal{C}_{\beta}\right\}$ is not complete, i.e., it cannot be used to represent arbitrary currents different from the stationary current. In this case the set $\left\{\mathcal{C}_{\beta}\right\}$ can be completed by adding further linear independent nonuniform cycles. The stationary current of these cycles is always zero.

Proof. Equipped with the decomposition in uniform cycles, we can now prove the affinity dependent bound (13) on the large deviation function. As a starting point we use, building on Ref. [16] and analogously to Ref. [8], the universally valid bound for the large deviation function of the distribution of the vector $\boldsymbol{J}=\left(J_{i j}\right)$ of all fluctuating currents along edges,

$$
I(\boldsymbol{J}) \leq \sum_{i<j}\left[J_{i j}\left(\operatorname{arsinh} \frac{J_{i j}}{a_{i j}}-\operatorname{arsinh} \frac{J_{i j}^{\mathrm{s}}}{a_{i j}}\right)-\left(\sqrt{a_{i j}^{2}+J_{i j}^{2}}-\sqrt{a_{i j}^{2}+\left(J_{i j}^{\mathrm{s}}\right)^{2}}\right)\right],
$$

with $a_{i j} \equiv 2 \sqrt{p_{i}^{\mathrm{s}} p_{j}^{\mathrm{s}} k_{i j} k_{j i}}$. This relation holds for all values of $\boldsymbol{J}$ that are consistent with Kirchhoff's law (8), otherwise $I(\boldsymbol{J})=\infty$. Eq. (19) follows from an exact expression 
for the "level 2.5" large deviation function $I(\boldsymbol{J}, \boldsymbol{p})$ for the joint distribution of the fluctuating current $\boldsymbol{J}$ and the fluctuating density $\boldsymbol{p}=\left(p_{i}\right)[16,17]$. By using the contraction principle, the large deviation function for the currents can be expressed as $I(\boldsymbol{J})=\min _{\boldsymbol{p}} I(\boldsymbol{J}, \boldsymbol{p})=I\left(\boldsymbol{J}, \boldsymbol{p}^{*}(\boldsymbol{J})\right)$. Using the stationary distribution $\boldsymbol{p}^{\mathbf{s}}$ instead of the most likely density $\boldsymbol{p}^{*}(\boldsymbol{J})$, one obtains as an upper bound on $I(\boldsymbol{J})$ the function $I\left(\boldsymbol{J}, \boldsymbol{p}^{\mathbf{s}}\right)$, which can be brought to the form of the r.h.s. of Eq. (19). The choice $\boldsymbol{p}^{\mathbf{s}}$ ensures that the bound is saturated for the most likely current $\boldsymbol{J}=\boldsymbol{J}^{\mathbf{s}}$.

At first, we assume for simplicity that the stationary current $J_{i j}^{\mathrm{s}}$ is non-zero along all edges with $k_{i j}>0$. Then, we can rewrite the bound (19) as

$$
I(\boldsymbol{J}) \leq \sum_{i<j} J_{i j}^{\mathrm{s}} \psi\left(J_{i j} / J_{i j}^{\mathrm{s}}, f_{i j}\right),
$$

where the function $\psi(\zeta, f)$ is defined as

$$
\psi(\zeta, f) \equiv \zeta \operatorname{arsinh}(\zeta / b)-\zeta f / 2-\sqrt{b^{2}+\zeta^{2}}+\sqrt{b^{2}+1}
$$

with

$$
b \equiv[\sinh (f / 2)]^{-1} .
$$

The function $\psi(\zeta, f)$ is the large deviation function for the current in an asymmetric random walk on a ring with uniform transition rates, with affinity per step $f$, and with stationary current $J^{\mathrm{s}}=1[1]$. For this highly symmetric network, there is only one possible current $J$ and $p_{i}^{\mathrm{s}}=p_{i}^{*}(J)=1 / N$ holds independently of $J$, so that Eq. (20) becomes an equality. For positive $f$ and fixed $\zeta, \psi(\zeta, f)$ is concave in $f$, moreover, $\psi(\zeta, f) / f$ decreases monotonically with increasing $f$, as proven in Appendix A and Appendix B, respectively. These essential properties are demonstrated in Fig. 3. The Legendre transform of $\psi(\zeta, f)$ is

$$
\lambda(y, f) \equiv \max _{\zeta}[y \zeta-\psi(\zeta, f)]=[\cosh (y+f / 2)-\cosh (f / 2)] / \sinh (f / 2) .
$$

Using the decomposition in the uniform cycles $\left\{\mathcal{C}_{\beta}\right\}$ from Eq. (18), for which all $f_{i j}$ along a cycle become positive, we can write Eq. (20) as

$$
I(\boldsymbol{J}) \leq \sum_{\beta} \mathcal{J}_{\beta}^{\mathrm{s}} \sum_{(i, j) \in \mathcal{C}_{\beta}} \psi\left(J_{i j} / J_{i j}^{\mathrm{s}}, f_{i j}\right) .
$$

Next, we set $J_{i j}=\xi J_{i j}^{\mathrm{s}}$ for all $i, j$. This choice simplifies the following steps and ensures that the fluctuating currents $J_{i j}$ are consistent with Kirchhoff's law (8). Having ensured via the uniform cycle decomposition that all $f_{i j}$ are positive, we can apply Jensen's inequality to the second sum in Eq. (24), in which the summation index runs over the $n_{\beta}$ edges of the cycle $\mathcal{C}_{\beta}$. Since the $\mathcal{J}_{\beta}^{\mathrm{s}}$ are positive, this procedure leads to

$$
I\left(\xi \boldsymbol{J}^{\mathrm{s}}\right) \leq \sum_{\beta} \mathcal{J}_{\beta}^{\mathrm{s}} n_{\beta} \sum_{(i, j) \in \mathcal{C}_{\beta}} \frac{1}{n_{\beta}} \psi\left(\xi, f_{i j}\right) \leq \sum_{\beta} \mathcal{J}_{\beta}^{\mathrm{s}} \mathcal{A}_{\beta}\left(n_{\beta} / \mathcal{A}_{\beta}\right) \psi\left(\xi, \mathcal{A}_{\beta} / n_{\beta}\right),
$$

where we have used Eq. (10). For a coarser bound, we identify the uniform cycle with minimal $\mathcal{A}_{\beta} / n_{\beta} \equiv \mathcal{A}^{*} / n^{*}$, which is positive as explained below Eq. (15). Using the monotonic decrease of $\psi(\zeta, f) / f$, the positivity of $\mathcal{J}_{\beta}^{\mathrm{s}} \mathcal{A}_{\beta}$, and Eq. (11), we obtain

$$
I\left(\xi \boldsymbol{J}^{\mathrm{s}}\right) \leq \sigma\left(n^{*} / \mathcal{A}^{*}\right) \psi\left(\xi, \mathcal{A}^{*} / n^{*}\right) .
$$



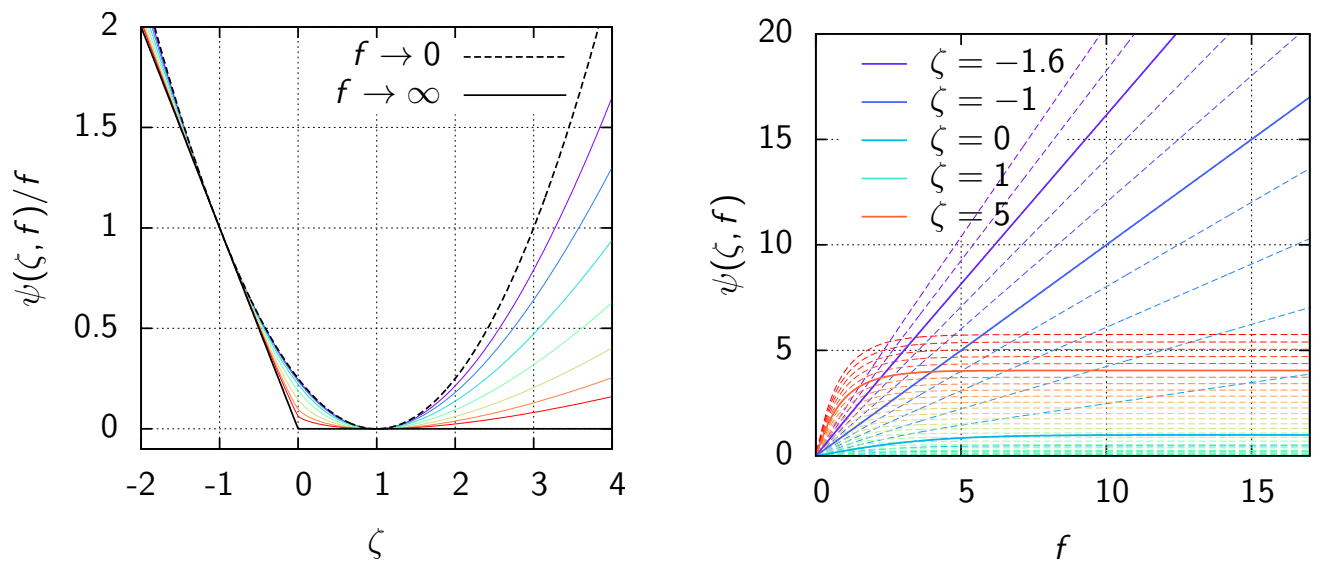

Figure 3. Left: The function $\psi(\zeta, f) / f$ as a function of $\zeta$ for values of $f$ ranging from 1 (violet) to $10^{1.2}$ (red) with logarithmic spacing. For fixed $\zeta$ and $f>0$, $\psi(\zeta, f) / f$ decreases monotonically in $f$, as required in Eq. (26). The limiting curves for $\lim _{f \rightarrow \infty} \psi(\zeta, f) / f=(|\zeta|-\zeta) / 2$ and for $\lim _{f \rightarrow 0} \psi(\zeta, f) / f=(\zeta-1)^{2} / 4$ are shown in black. Right: The function $\psi(\zeta, f)$ as a function of $f$ for values of $\zeta$ ranging from -2 (violet) to 6 (red). Selected values of $\zeta$ are shown as solid lines, in particular $\psi(-1, f)=f$ and $\psi(1, f)=0$. Obviously, $\psi(\zeta, f)$ is concave for $f \geq 0$.

Finally, we adopt a more elegant formulation of the large deviation function taking the cycle currents $\mathcal{J}_{\beta}$ as argument instead of $\left(J_{i j}\right)=\boldsymbol{J}$, i.e., we write

$$
\mathcal{I}(\mathcal{J}) \equiv I\left(\sum_{\beta} \mathcal{J}_{\beta} \chi^{\beta}\right) .
$$

We thus avoid currents that are inconsistent with Kirchhoff's law, for which the large deviation function would become infinite. The scaled cumulant generating function corresponding to the large deviation function (27) turns out to be bounded by

$$
\begin{aligned}
\lambda(\boldsymbol{z}) & =\max _{\mathcal{J}}\left[\sum_{\beta} z_{\beta} \mathcal{J}_{\beta}-\mathcal{I}(\mathcal{J})\right] \\
& \geq \max _{\mathcal{J}=\xi \mathcal{J}^{\mathrm{s}}}\left[\boldsymbol{z} \cdot \mathcal{J}^{\mathrm{s}} \xi-I\left(\xi \boldsymbol{J}^{\mathrm{s}}\right)\right] \\
& \geq \max _{\xi}\left[\boldsymbol{z} \cdot \mathcal{J}^{\mathrm{s}} \xi-\sigma\left(n^{*} / \mathcal{A}^{*}\right) \psi\left(\xi, \mathcal{A}^{*} / n^{*}\right)\right] \\
& =\frac{\sigma}{\mathcal{A}^{*} / n^{*}} \lambda\left(\boldsymbol{z} \cdot \mathcal{J}^{\mathrm{s}}\left(\mathcal{A}^{*} / n^{*}\right) / \sigma, \mathcal{A}^{*} / n^{*}\right) \\
& =\sigma \frac{\cosh \left[\left(\boldsymbol{z} \cdot \mathcal{J}^{\mathrm{s}} / \sigma+1 / 2\right) \mathcal{A}^{*} / n^{*}\right]-\cosh \left[\mathcal{A}^{*} /\left(2 n^{*}\right)\right]}{\left(\mathcal{A}^{*} / n^{*}\right) \sinh \left[\mathcal{A}^{*} /\left(2 n^{*}\right)\right]} \\
& \equiv B\left(\boldsymbol{z} \cdot \mathcal{J}^{\mathrm{s}}, \mathcal{A}^{*} / n^{*}, \sigma\right),
\end{aligned}
$$

where we have used Eq. (26) for the third line and Eq. (23) for the fifth line. Although the uniform cycle decomposition was essential in the steps leading to this result, its final statement is no longer dependent on the specific choice of independent cycles, as can be seen from the following argument. In a different complete set of cycles the 
cycle currents are represented as $\tilde{\mathcal{J}}_{\alpha}=\sum_{\beta} C_{\alpha \beta} \mathcal{J}_{\beta}$ with some transformation matrix $C_{\alpha \beta}$. The corresponding scaled cumulant generating function then reads

$\tilde{\lambda}(\tilde{\boldsymbol{z}})=\lim _{t \rightarrow \infty} \frac{1}{t} \log \left\langle\exp \left[\sum_{\alpha} \tilde{z}_{\alpha} \tilde{\mathcal{J}}_{\alpha} t\right]\right\rangle=\lim _{t \rightarrow \infty} \frac{1}{t} \log \left\langle\exp \left[\sum_{\alpha, \beta} \tilde{z}_{\alpha} C_{\alpha \beta} \mathcal{J}_{\beta} t\right]\right\rangle=\lambda\left(C^{\top} \tilde{\boldsymbol{z}}\right)$

and satisfies the bound

$$
\tilde{\lambda}(\tilde{\boldsymbol{z}}) \geq B\left(C^{\top} \tilde{\boldsymbol{z}} \cdot \mathcal{J}^{\mathbf{s}}, \mathcal{A}^{*} / n^{*}, \sigma\right)=B\left(\tilde{\boldsymbol{z}} \cdot \tilde{\mathcal{J}}^{\mathrm{s}}, \mathcal{A}^{*} / n^{*}, \sigma\right),
$$

which finally proves Eq. (13) for arbitrary cycle decompositions. It should be noted that, a priori, $\mathcal{A}^{*} / n^{*}$ still refers to the minimal $\mathcal{A}_{\beta} / n_{\beta}$ in a set of uniform cycles. However, in a possible application, where the network topology and the cycle affinities are known but individual transition rates are not known, the more general result (13), where $\mathcal{A}^{*} / n^{*}$ refers to the minimum over all cycles, might lead to a weaker bound that is more useful. Since uniform cycles always have affinity $\mathcal{A}_{\beta}>0$, the minimization can be restricted to cycles with positive affinity. Thus, in the physically important case of a network containing cycles with zero affinity, Eq. (13) provides a bound that is stronger than the parabolic bound that is obtained for $\mathcal{A}^{*} / n^{*} \rightarrow 0$. This fact is in accordance with the insight from Ref. [18], stating that coarse graining such cycles with zero affinity has little effect on the fluctuations of the entropy production.

Vanishing currents and equilibrium. So far, we have considered only the case of nonvanishing stationary currents $J_{i j}^{\mathrm{s}}$ along all edges. If this is not the case for some edges (although $k_{i j}>0$ ), we have to split the bound for the large deviation function in Eq. (19) in two parts as

$$
I(\boldsymbol{J}) \leq \sum_{i<j \mid J_{i j}^{\mathrm{s}} \neq 0} J_{i j}^{\mathrm{s}} \psi\left(J_{i j} / J_{i j}^{\mathrm{s}}, f_{i j}\right)+\sum_{i<j \mid J_{i j}^{\mathrm{s}}=0}\left[J_{i j} \operatorname{arsinh} \frac{J_{i j}}{a_{i j}}-\sqrt{a_{i j}^{2}+J_{i j}^{2}}+a_{i j}\right] .
$$

Evaluating this bound along currents $\boldsymbol{J}=\xi \boldsymbol{J}^{\mathbf{s}}$, the second sum vanishes while the first sum leads along the same lines as before to the bound (28). However, in the equilibrium case, where all stationary currents vanish, this procedure leads merely to the trivial statement $\lambda(\boldsymbol{z}) \geq 0$. While typical fluctuations in equilibrium systems can be well described within linear response theory, rare fluctuations exhibit many features akin to systems far from equilibrium [19]. A non-trivial bound for these rare fluctuations in equilibrium systems, similar to the one derived in Ref. [5] for unicyclic networks, is obtained by letting the affinity $\mathcal{A}_{\alpha}$ of a single fundamental cycle go to zero while keeping the affinities of the other fundamental cycles fixed at zero. Then, none of the cycles can have an affinity smaller than $\mathcal{A}_{\alpha}$, so that $\mathcal{A}^{*}=\mathcal{A}_{\alpha}$. The length of the relevant cycle $n^{*}$ can be bounded by the total number of states $N \geq n^{*}$. Due to the Einstein relation [20], the stationary current $\mathcal{J}_{\alpha}^{\mathrm{s}}$ is given for small $\mathcal{A}_{\alpha}$ by

$$
\mathcal{J}_{\alpha}^{\mathrm{s}}=D_{\alpha} \mathcal{A}_{\alpha}+\mathcal{O}\left(\mathcal{A}_{\alpha}^{2}\right),
$$

where $D_{\alpha}$ is the diffusion coefficient

$$
2 D_{\alpha} \equiv \lim _{t \rightarrow \infty} t\left\langle\left(\mathcal{J}_{\alpha}-\mathcal{J}_{\alpha}^{\mathrm{s}}\right)^{2}\right\rangle .
$$


With the entropy production (11) reducing to $\sigma=\mathcal{J}_{\alpha}^{\mathrm{s}} \mathcal{A}_{\alpha}=D_{\alpha} \mathcal{A}_{\alpha}^{2}$, we thus obtain as bound on the scaled cumulant generating function for the equilibrium fluctuations of $\mathcal{J}_{\alpha}$

$$
\lambda_{\alpha}(z) \geq \lim _{\mathcal{A}_{\alpha} \rightarrow 0} B\left(z D_{\alpha} \mathcal{A}_{\alpha}, \mathcal{A}_{\alpha} / N, D_{\alpha} \mathcal{A}_{\alpha}^{2}\right)=2 N^{2} D_{\alpha}(\cosh (z / N)-1) .
$$

This bound is saturated for $z \ll 1$, where $\lambda_{\alpha}(z)$ depends quadratically on $z$ according to the Gaussian distribution of typical fluctuations within linear response. The probability of extreme fluctuations beyond linear response deviates the more from this Gaussian shape the smaller the number of states in the network is.

Conclusion. We have shown that for every Markov network it is possible to construct a set of independent cycles such that all affinities and stationary cycle currents are positive and that all stationary currents along edges are aligned with the direction of the cycles. We call this a decomposition in uniform cycles. Based on such a decomposition of the stationary currents, we have proven the hyperbolic cosine shaped bound on the scaled cumulant generating function for current fluctuations (13), which we have previously conjectured in Ref. [5]. This bound refines the parabolic bound previously proven in Ref. [8] and requires knowledge of the minimal positive affinity per number of edges in any cycle of the network. The bound is universally valid for arbitrary currents in arbitrary networks and entails a bound on the Fano factor in enzyme kinetics, previously conjectured in Ref. [10], which is thereby proven as well. We have also proven a new universal bound on equilibrium fluctuations, given in Eq. (34), that depends only on the diffusion coefficient and on the number of states.

\section{Appendix A. Proof of the concavity of $\psi(\zeta, f)$ in $f$}

The derivative of $\psi(\zeta, f)$ in Eq. (21) can be written as

$$
\partial_{f} \psi(\zeta, f)=\frac{1}{2} \sqrt{b^{2}+\zeta^{2}} \sqrt{b^{2}+1}-\frac{b^{2}}{2}-\frac{\zeta}{2} .
$$

As a function of $b=[\sinh (f / 2)]^{-1}$ (which decreases monotonically in $f$ ), this expression increases monotonically for $b>0$, since

$$
\partial_{b} \partial_{f} \psi(\zeta, f)=\frac{b}{2}\left(\sqrt{\frac{b^{2}+\zeta^{2}}{b^{2}+1}}+\sqrt{\frac{b^{2}+1}{b^{2}+\zeta^{2}}}-2\right) \geq 0
$$

(note that $x+1 / x \geq 2$ for $x>0$ ). Therefore, $\partial_{f} \psi$ decreases monotonically in $f$ and

$$
\partial_{f}^{2} \psi(\zeta, f)<0
$$

holds for all $\zeta \in \mathbb{R}$ and $f>0$.

\section{Appendix B. Proof of the monotonic decrease of $\psi(\zeta, f) / f$ in $f$}

The monotonic decrease of $\psi(\zeta, f) / f$ in $f$ for $f>0$ is equivalent to the monotonic increase of its Legendre transform

$$
\mu(z, f) \equiv \max _{\zeta}[\zeta z-\psi(\zeta, f) / f]=\lambda(z f, f) / f=\frac{\cosh [(z+1 / 2) f]-\cosh (f / 2)}{f \sinh (f / 2)} \equiv \frac{A(z, f)}{B(f)} .
$$


The derivative $\partial_{f} \mu(z, f)$ is non-negative if

$$
\begin{aligned}
& 0 \leq C(z) \equiv \partial_{f} A(z, f) B(f)-A(f) \partial_{f} B(f) \\
&=[(z+1 / 2) \sinh ((z+1 / 2) f)-(1 / 2) \sinh (f / 2)] f \sinh (f / 2) \\
& \quad-[\cosh ((z+1 / 2) f)-\cosh (f / 2)][\sinh (f / 2)+(f / 2) \cosh (f / 2)] .
\end{aligned}
$$

Equating $\partial_{z} C(z)$ to zero leads to

$$
2(z+1 / 2) \tanh (f / 2)=\tanh [(z+1 / 2) f],
$$

which has the three solutions $z=-1,-1 / 2,0$. The stationary points $z=0$ and $z=-1$ are minima since

$$
\partial_{z}^{2} C(z=0)=\frac{f^{2}}{2}(\sinh f-f)>0
$$

and $C(z)$ is symmetric with respect to $-1 / 2$. Thus, the center of symmetry at $z=-1 / 2$ is a maximum of $C(z)$ and the global minimum of $C(z)$ is given by $C(0)=C(-1)=0$.

\section{References}

[1] J. L. Lebowitz and H. Spohn, "A Gallavotti-Cohen-type symmetry in the large deviation functional for stochastic dynamics," J. Stat. Phys. 95 (1999) 333.

[2] D. Andrieux and P. Gaspard, "Fluctuation theorem for currents and Schnakenberg network theory," J. Stat. Phys. 127 (2007) 107.

[3] M. Polettini and M. Esposito, "Transient fluctuation theorems for the currents and initial equilibrium ensembles," J. Stat. Mech. (2014) P10033.

[4] U. Seifert, "Stochastic thermodynamics, fluctuation theorems, and molecular machines," Rep. Prog. Phys. 75 (2012) 126001.

[5] P. Pietzonka, A. C. Barato, and U. Seifert, "Universal bounds on current fluctuations," Phys. Rev. E 93 (2016) 052145.

[6] R. S. Ellis, Entropy, Large Deviations, and Statistical Mechanics. Springer-Verlag, Berlin, 2006.

[7] H. Touchette, "The large deviation approach to statistical mechanics," Phys. Rep. 478 (2009) 1.

[8] T. R. Gingrich, J. M. Horowitz, N. Perunov, and J. L. England, "Dissipation bounds all steady-state current fluctuations," Phys. Rev. Lett. 116 (2016) 120601.

[9] A. C. Barato and U. Seifert, "Thermodynamic uncertainty relation for biomolecular processes," Phys. Rev. Lett. 114 (2015) 158101.

[10] A. C. Barato and U. Seifert, "Universal bound on the fano factor in enzyme kinetics," J. Phys. Chem. B 119 (2015) 6555.

[11] J. Schnakenberg, "Network theory of microscopic and macroscopic behavior of master equation systems," Rev. Mod. Phys. 48 (1976) 571.

[12] T. L. Hill, Free Energy Transduction and Biochemical Cycle Kinetics. 2nd ed., Dover, Mineola, New York, 1989

[13] H. Qian, "Phosphorylation energy hypothesis: Open chemical systems and their biological functions," Annu. Rev. Phys. Chem. 58 (2007) 113.

[14] J. MacQueen, "Circuit processes," Ann. Prob. 9 (1981) 604.

[15] B. Altaner, S. Grosskinsky, S. Herminghaus, L. Katthän, M. Timme, and J. Vollmer, "Network representations of nonequilibrium steady states: Cycle decompositions, symmetries, and dominant paths," Phys. Rev. E 85 (2012) 041133.

[16] C. Maes and K. Netočný, "Canonical structure of dynamical fluctuations in mesoscopic nonequilibrium steady states," EPL 82 (2008) 30003.

[17] A. C. Barato and R. Chetrite, "A formal view on level 2.5 large deviations and fluctuation relations," J. Stat. Phys. 160 (2015) 1154.

[18] A. Puglisi, S. Pigolotti, L. Rondoni, and A. Vulpiani, "Entropy production and coarse graining in Markov processes," J. Stat. Mech. (2010) P05015. 
[19] S. Rahav and C. Jarzynski, "Nonequilibrium fluctuation theorems from equilibrium fluctuations," New J. Phys. 15 (2013) 125029.

[20] D. Andrieux and P. Gaspard, "A fluctuation theorem for currents and non-linear response coefficients," J. Stat. Mech. (2007) P02006. 